\title{
8 Pole High Temperature Superconductor Microstrip Dual Band Bandpass Filter Design
}

\author{
Kenneth S. K. Yeo ${ }^{1}$ and Michael J. Lancaster ${ }^{2}$ \\ ${ }^{1}$ School of Computing, IT and Engineering, University of East London, London E16 2RD, UK \\ ${ }^{2}$ Electronic, Electrical and Computing Eng., The University of Birmingham, Birmingham B15 2TT, UK
}

\begin{abstract}
Dual-band filters are normally used for filtering two frequency bands that are not too close together. However, this paper presents a HTS dual-band bandpass filter that can be used to achieve isolation between two frequency bands that are only a few tens of $\mathrm{MHz}$ apart. Transmission zeros are placed in between the two frequency bands using electromagnetic coupling between non-adjacent resonators which result in high isolation between the two bands. The simulation and experimental results of a High Temperature Superconductor dual-band bandpass filter with very narrow bandwidth will be presented here.
\end{abstract}

Index Terms - Bandpass, dual-band, filter, HTS, microstrip, superconductor.

\section{INTRODUCTION}

Dual-band bandpass filters have received must attention in recent years. This is mainly due to the rapid evolving wireless communication technology, and also the ever increasing demand for personal/consumer communication applications.

Dual-band bandpass filters can be divided into two categories. Category I is where the frequency separation between the two bands is small and category II is where the frequency separation between the two bands is large. Dualband bandpass filters in category I are particularly useful for rejecting unwanted frequency spectrum that falls in between a wanted band of the frequency spectrum. Whereas dual-band bandpass filters in category II are useful for communication system that employs multiple frequency spectrum bands, e.g. Wireless LAN at $2.4 \mathrm{GHz}$ and $5.2 \mathrm{GHz}$. For category I application, conventionally, it is achieved by cascading a bandpass filter with a bandstop filter [1]. With the advancement in the filter design theories, a dual-band bandpass filter can be achieved by placing transmission zeros into the passband of a wider-bandwidth bandpass filter to achieve a stopband within the passband. This type of dualband bandpass filter is achieved by cross-coupling arrangement between synchronously tuned resonators. Although theoretically there is no reason why a cross-coupled dual-band filter cannot be achieved in category II, in practice the coupling strength requirements are normally too strong to be realized. Category II dual-band bandpass filters are normally achieved by employing the harmonic of the fundamental resonator mode [2], using quarter-wavelength admittance inverters between resonators [3] instead of electromagnetic coupling or even using cross-coupled asynchronously tuned resonators [4].
In this paper, the category I dual-band filter will be presented based on coupling of synchronously tuned resonators. The bandwidth of the two individual bandpass filter is $10 \mathrm{MHz}$ with a fractional bandwidth of approximately $0.5 \%$. The fractional bandwidth of the dual-band is $1.6 \%$, i.e. $\left(f_{\mathrm{o}, \mathrm{BPU}}-f_{\mathrm{o}, \mathrm{BPL}}\right) / f_{\mathrm{o}}[3]$. The circuit model and electromagnetic simulations together with cryogenic measurement will be presented.

\section{DUAL-BAND FILTER DESIGN}

The dual-band filter presented in this paper consists of 8 resonators. The filter will have 8 poles where 4 poles are located on each passband. In addition, the filter will also exhibit 4 transmission zeros, a pair of transmission zero for each passband.

Fig. 1 shows the configuration of all the resonators and the coupling layout of the dual-band bandpass filter. The dualband characteristic is achieved by having weaker coupling strength between the resonators 4 and 5 in comparison to the coupling strength between the resonators 3 and 6 . In a single band bandpass filter, the coupling between the resonators 4 and 5 must be stronger than the coupling between the resonators 3 and 6 .

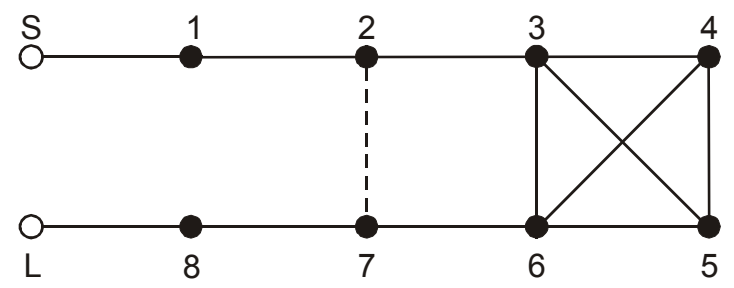

Fig. 1. Dual-band bandpass filter resonators and the coupling configuration (coupling: slash line is $180^{\circ}$ phase different compared to solid line and black dot $=$ resonator)

The specification of the designed dual-band bandpass filter is

Center frequency, $f_{\mathrm{o}}$ :

Center frequency of lower passband, $f_{\mathrm{o}, \mathrm{BPL}}$ :

Center frequency of upper passband, $f_{\mathrm{o}, \mathrm{BPU}}$ :

$1975.5 \mathrm{MHz}$

Bandwidth of lower passband, $\mathrm{BW}_{\mathrm{L}}$ :

$1960 \mathrm{MHz}$

$1991 \mathrm{MHz}$

$10 \mathrm{MHz}$

Bandwidth of upper passband, $\mathrm{BW}_{\mathrm{U}}$ : 
A circuit model of the dual-band filter is formulated and simulated using Agilent Advanced Design Systems (ADS). All the 8 resonators are modeled using parallel inductor, $L$, and capacitor, $C$, which has a value of $5.77 \times 10^{-4} \mathrm{nH}$ and $1.1241 \times 10^{4} \mathrm{pF}$, respectively. The coupling between resonators is modeled using admittance inverters ( $\mathrm{J}$-inverters) in the form of PI-capacitors $[5,6]$. The J-inverter values are

$$
\begin{array}{ll}
J_{12}=J_{78}=2.396 & J_{36}=0.650 \\
J_{23}=J_{67}=1.142 & J_{27}=-0.148 \\
J_{34}=J_{56}=2.028 & J_{35}=J_{46}=0.006 \\
J_{45}=0.392 & J_{01}=J_{89}=1.239
\end{array}
$$

The corresponding coupling coefficients can be determined using Eqn. (5) in [5] and external quality factor can be determined using Eqn. (1) and the values are

$$
\begin{array}{ll}
M_{12}=M_{78}=0.01717 & M_{36}=0.00466 \\
M_{23}=M_{67}=0.00818 & M_{27}=-0.00106 \\
M_{34}=M_{56}=0.01454 & M_{45}=0.00281 \\
M_{35}=M_{46}=0.000045 & Q_{\text {ext }}=113
\end{array}
$$

$Q_{\text {ext }}=\frac{\omega_{o} C}{J_{m, m+1}}$ where $m=0$ or $n$ (the order of the filter)

\section{MICROSTRIP DESIGN}

The microstrip dual-band bandpass filter uses folded hairpin resonator topology, which is shown in Fig. 2, as the building block. From the coupling layout shown in Fig. 1, an in-phase cross coupling is required, i.e. $\mathrm{M}_{36}$ and $\mathrm{M}_{45}$ are in-phase. To achieve in-phase cross coupling for microstrip resonators, with at least one symmetrical axis (shown in Fig. 3) the resonators have to be arranged in a similar fashion as those shown in Fig. 4.

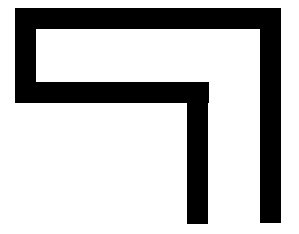

Fig. 2. Folder hairpin resonator.

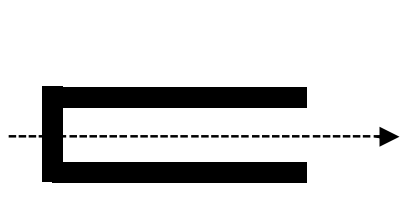

Hairpin Resonator

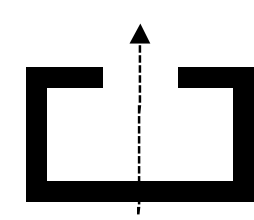

Open Loop Resonator

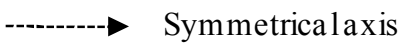

Fig. 3. Microstrip resonator with at least one symmetrical axis.

With close inspection, one will notice that unwanted couplings between the resonators 3 and 5 and the resonators 4 and 6 will exist in that arrangement. In comparison to the arrangement for out-of-phase cross coupling, which is often used to introduce a pair of transmission zeros in cascaded quadruplet filters [6], the unwanted coupling between the resonators 3 and 5 and the resonators 4 and 6 are much weaker. This can be explained based on mixed coupling. The coupling structure between the resonators 3 and 5 in an inphase cross coupling arrangement, will exhibit a type-I mixed coupling, where the electric and magnetic couplings enhance each other [5]. Whereas in out-of-phase cross-coupling arrangement, the coupling between the resonators 3 and 5 and the resonators 4 and 6 will exhibit type-II mixed coupling where the electric and magnetic couplings cancelling each other out [6]. As a result, for the same coupling distance, the coupling between the resonators 3 and 5 and the resonators 4 and 6 are much stronger in an in-phase cross-coupling structure compared to an out-of-phase cross-coupling structure. Therefore, the unwanted coupling has a much stronger effect in distorting the filter characteristic in an inphase cross-coupling structure.

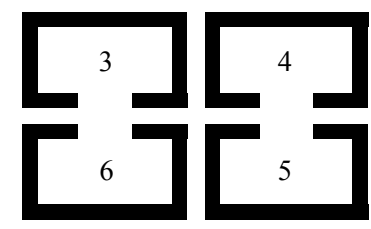

Open Loop Resonators

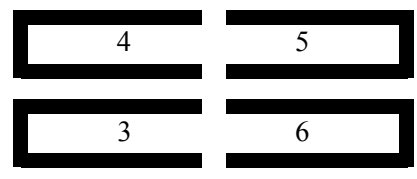

Hairpin Resonators
Fig. 4. Arrangement of in-phase cross coupling ( $\mathrm{M}_{36}$ and $\mathrm{M}_{45}$ are in-phase) for resonator with one symmetrical axis.

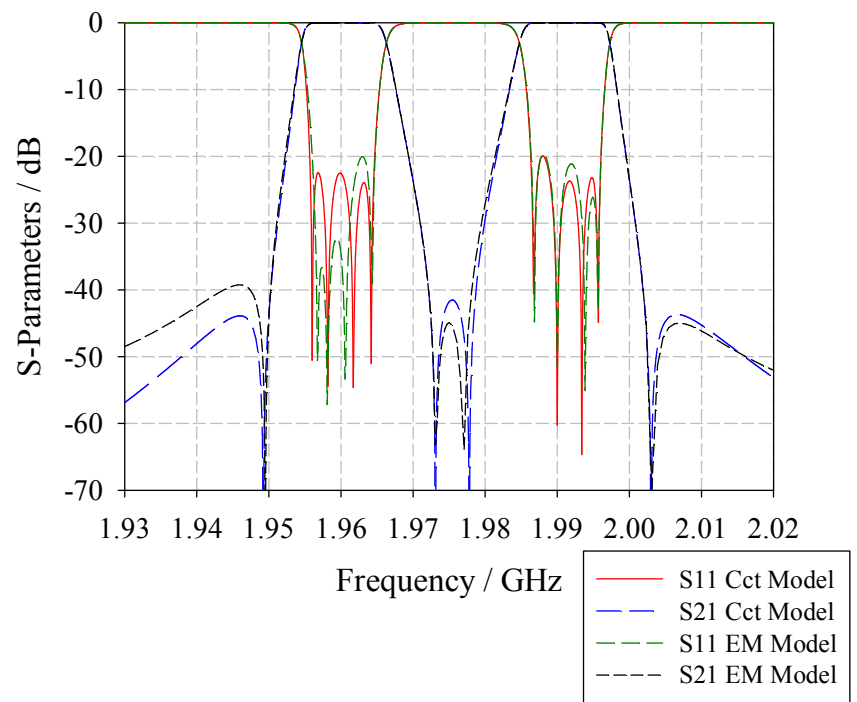

Fig. 5. Comparison of circuit model and EM simulation results

In order to reduce the unwanted cross-coupling, the folded hairpin resonator is introduced. By removing all the symmetries in the resonator structure, the resonators 3 and 5 and the resonators 4 and 6 can be placed further away in an in- 
phase cross-coupling arrangement. However, in a planar structure, coupling between resonators is always present no matter how far apart they are placed except in the type-II mixed coupling when the electric coupling and magnetic coupling is equal. In this design, the weak coupling of the resonators 3 and 5 and the resonators 4 and 6 are incorporated in the design.

The microstrip dual-band bandpass filter is simulated using Momentum of Agilent Advanced Design Systems. The simulation results are given in Fig. 5. In Fig. 5, the circuit model simulation results are also presented. The EM simulation results show a good agreement with the circuit model simulation results.

\section{CIRCUIT FABRICATION}

The high temperature superconductor (HTS) dual-band bandpass filter is fabricated using photolithographic process onto a single crystal Magnesium Oxide $(\mathrm{MgO})$ substrate with a thin film of Yttrium Barium Copper Oxide (YBCO) on both sides. The YBCO films have a thickness of $600 \mathrm{~nm}$ with $T_{c}$ of $87.7 \mathrm{~K}$ and $\mathrm{J}_{\mathrm{c}}$ of $2.4 \mathrm{MA} / \mathrm{cm}^{2}$. The $\mathrm{MgO}$ substrate has a thickness on $0.508 \mathrm{~mm}$ with dielectric constant of 9.8 and loss tangent in the region of $10^{-6}$.

The layout of the 8 pole dual-band bandpass filter ( 4 poles on each bandpass) with 4 transmission zeros is shown in Fig. 6 . The linewidth of the microstrip is $0.5 \mathrm{~mm}$ which is designed for $50 \Omega$.

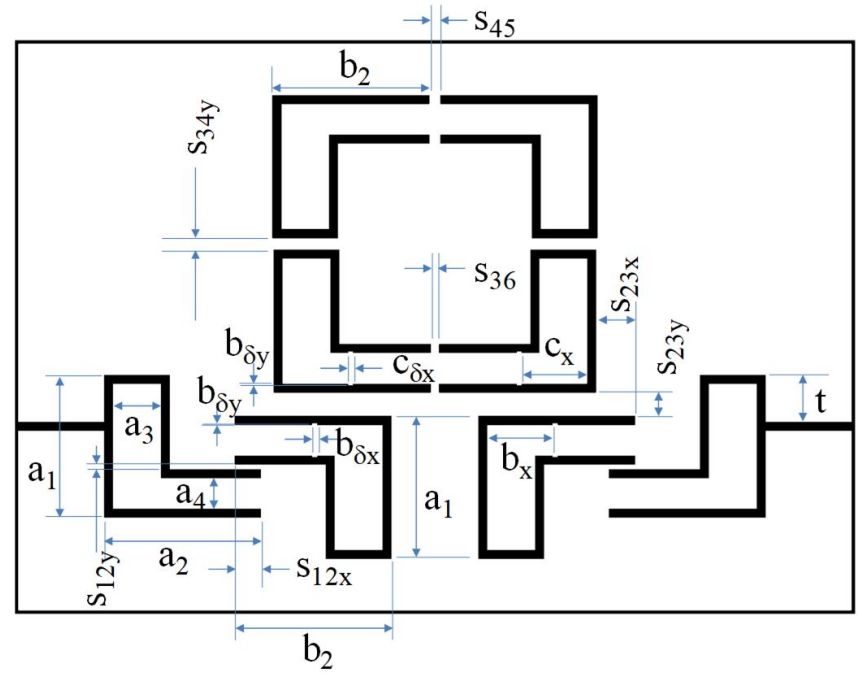

Fig. 6. Dual-band bandpass filter layout

The dimensions of the filter layout parameters corresponding to Fig. 6 are given in Table I. All the dimensions given in Table I are in $\mathrm{mm}$.

The fabricated HTS circuit is mounted on a gold plated titanium carrier. Titanium is used because of its thermal expansion coefficient which is relatively close to the thermal expansion coefficient of $\mathrm{MgO}$. This is important to ensure that the $\mathrm{MgO}$ will not crack due different expansion rate when cooling from $300 \mathrm{~K}$ down to $60 \mathrm{~K}$. The titanium carrier is then mounted on a brass housing fixed with K-connectors. Sliding contacts are used for connecting the K-connectors to the HTS microstrip input and output feed lines. Fig. 7 shows a photograph of the assembled HTS dual-band bandpass filter with its lid opened.

TABLE I

SUMMARY OF CIRCUIT LAYOUT PARAMETERS

\begin{tabular}{|c|c|c|c|c|c|c|}
\hline $\mathrm{a}_{1}$ & $a_{2}$ & $a_{3}$ & $a_{4}$ & $\mathrm{~b}_{2}$ & $b_{x}$ & $\mathrm{~b}_{\delta \mathrm{x}}$ \\
\hline 8.3 & 9.099 & 2.8 & 2.8 & 9.112 & 3.8 & 0.32 \\
\hline$c_{x}$ & $c_{\delta x}$ & $\mathrm{~S}_{12 \mathrm{x}}$ & $\mathrm{S}_{12 \mathrm{y}}$ & $\mathrm{S}_{23 \mathrm{x}}$ & $\mathrm{S}_{23 \mathrm{y}}$ & $\mathrm{S}_{34 \mathrm{y}}$ \\
\hline 3.8 & 0.3 & 0.3 & 1.5311 & 2.287 & 1.3805 & 0.6908 \\
\hline $\mathrm{S}_{45}$ & $\mathrm{~S}_{36}$ & $\mathrm{t}$ & & & & \\
\hline 0.648 & 0.4936 & 2.7349 & & & & \\
\hline
\end{tabular}

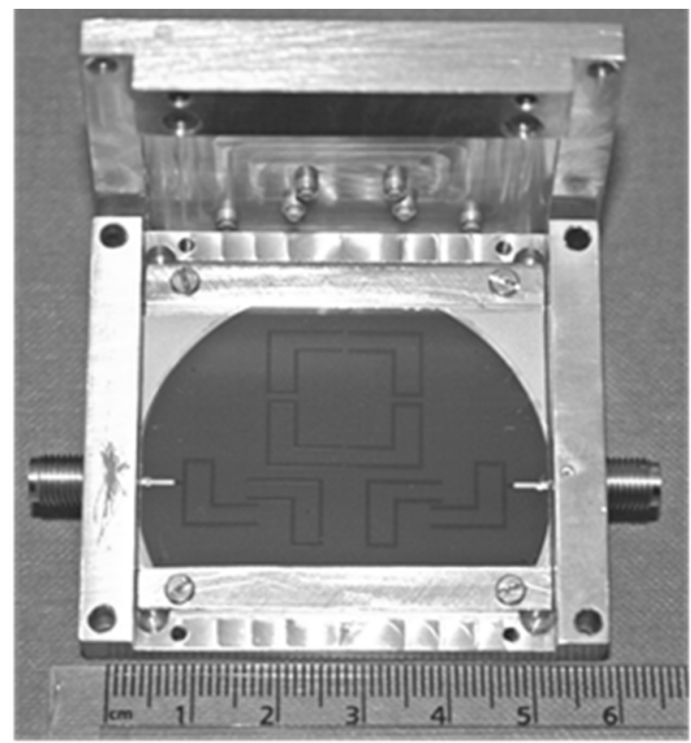

Fig. 7. Photograph of the assembled HTS dual-band passband filter

\section{EXPERIMENTAL RESULTS}

The assembled HTS dual-band bandpass filter is mounted onto the cold head of Sterling Cooler. The filter is cooled down to $60 \mathrm{~K}$ and the filter response is measured. Fig. 8 shows the filter response without any tuning performed. The measured results show good agreement with the EM simulated results. There is a shift of approximately $2.3 \mathrm{MHz}$ upward in the frequency response of the measured filter. This shift can be corrected/tuned using mechanical tuning screws [7]. However, this was not performed in this experiment. The measured ripple is also slightly greater than the simulated. This is mainly due to fabrication tolerance and variation of substrate thickness across the circuit.

The measured insertion loss shows gain of approximately $0.5 \mathrm{~dB}$. This is due to the calibration being performed at room 
temperature while the measurement was performed at $60 \mathrm{~K}$. Therefore, the reference point of the calibration has shifted. Fig. 9 shows a close up of the passband ripple. At approximately $1.953 \mathrm{GHz}$, the $\left|\mathrm{S}_{11}\right|$ is measured at approximately $0.23 \mathrm{~dB}$ which correspond to a transmission zero at the $\left|\mathrm{S}_{21}\right|$. If the calibration was performed at $60 \mathrm{~K}$, the $\left|\mathrm{S}_{11}\right|$ of this transmission zero is approximately at $0 \mathrm{~dB}$. Therefore, it is a safe hypothesis to assume that the $0 \mathrm{~dB}$ reference is very close to the return loss at $1.953 \mathrm{GHz}$. Using that as reference, the minimum filter passband insertion loss is estimated as $0.18 \mathrm{~dB}$. Fig. 9 shows a wideband measurement of the filter. There is no spurious mode measured between 1 $\mathrm{GHz}$ to $3 \mathrm{GHz}$ spectrum as shown in Fig. 10.

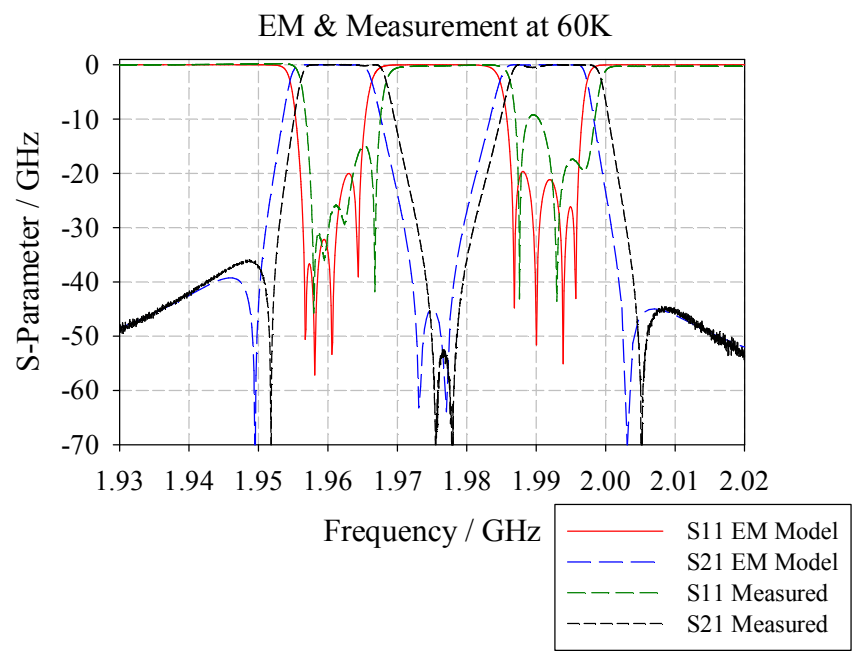

Fig. 8. Comparison of EM simulation and measurement results at $60 \mathrm{~K}$

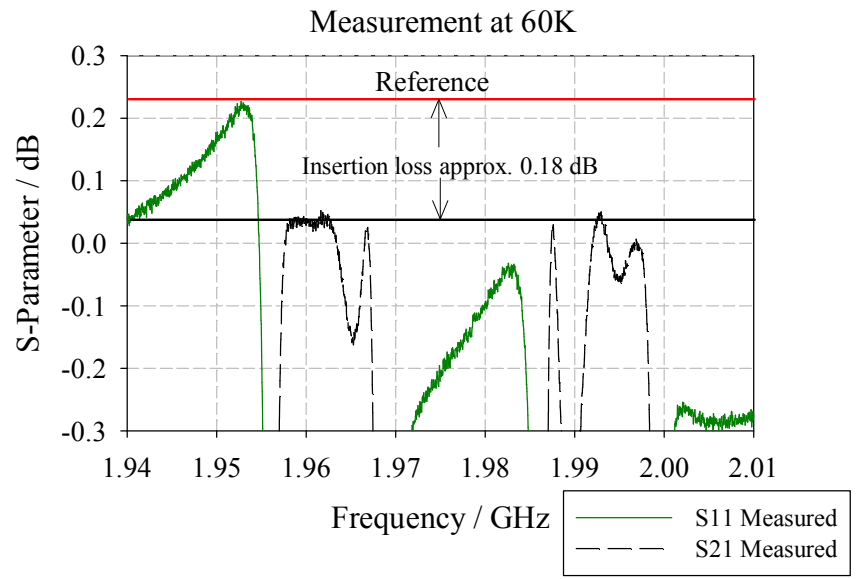

Fig. 9. Close-up response of the passband ripple at $60 \mathrm{~K}$

\section{Conclusion}

The simulation and experimental results of an 8 pole HTS dual-band bandpass filter have been presented. The experimental results are in good agreement with the design. Good isolation between the two passband has also been achieved in the experimental results. However, further improvement can be achieved by fine tuning the resonators centre frequencies. This can be done by using mechanical tuning screws which are mounted on the box lid. The loss of the filter is very small. The minimum passband insertion loss is estimated at approximately $0.18 \mathrm{~dB}$.

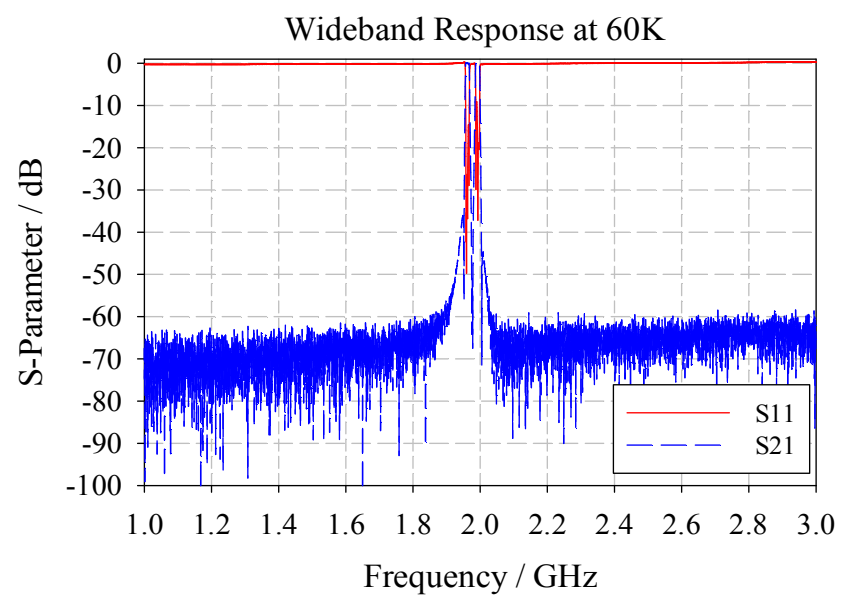

Fig. 10. Wideband measurement of the HTS filter at $60 \mathrm{~K}$

\section{ACKNOWLEDGEMENT}

This work was funded under EPSRC Portfolio grant GR/S60471/01. The authors would like to Clifford Ansell and Donna M. Holdom from the University of Birmingham for their technical assistance.

\section{REFERENCES}

[1] L. -C. Tsai and C. -W. Hsue, "Dual-Band Bandpass Filters Using Equal-Length Coupled-Serial-Shunted Lines and ZTransform Technique," IEEE Trans. Microwave, Theory \& Tech., vol. 52, no. 4, pp. 1111-1117, April 2004.

[2] S. -F. Cheng, Y. -L. Jeng, and J. -L. Chen, "Dual-Band StepImpedance Bandpass Filter for Multimode Wireless LANs," Electronics Letters, vol. 40, no. 1, pp. 38-39, 2004.

[3] X. Guan, Z. Ma, P. Cai, Y. Kobayashi, T. Anada and G. Hagiware, "Synthesis of Dual-Band Bandpass Filters Using Successive Frequency Transformation and Circuit Conversions," IEEE Microwave \& Wireless Components letters, vol. 16 , no. 3, 2006.

[4] J. -T. Kuo, H. -S. Cheng, "Design of Quasi-Elliptic Function Filters With a Dual-Passband Response," IEEE Microwave \& Wireless Components Letters, vol. 14, no. 10, 2004.

[5] K. S. K. Yeo, M. J. Lancaster and J. -S. Hong, "The Design of Microstrip Six-Pole Quasi-Elliptic Filter with Linear Phase Response Using Extracted-Pole Technique," IEEE Trans. Microwave Theory \& Tech., vol. 49, no. 2, pp 321-327, 2002.

[6] J. -S. Hong and M. J. Lancaster, "Couplings of Microstrip Square Open-Loop Resonators for Cross-Coupled Planar Microwave Filters," IEEE Trans. Microwave Theory \& Tech., vol. 44, no. 12, pp. 2099-2108, 1996.

[7] J.-S Hong, M. J. Lancaster, D. Jedamzik and R. B. Greed, “On the Development of Superconducting Microstrip Filters for Mobile Communications Applications", IEEE Trans. Microwave Theory \& Tech., vol. 47, no. 9, pp. 1656-1663, 1999. 